\title{
Gexplora - user interface that highlights and explores the density of genomic elements along a chromosomal sequence
}

Thomas Nussbaumer, Olivia Debnath, Parviz Heidari

\begin{abstract}
The density of genomic elements such as genes or transposable elements along its consecutive sequence can provide an overview of a genomic sequence while in the detailed analysis of candidate genes it may depict enriched chromosomal hotspots harbouring genes that explain a certain trait. The herein presented python-based graphical user interface Gexplora allows to obtain more information about a genome by considering sequence-intrinsic information from external databases such as EnsembI, OMA and STRING database using REST API calls to retrieve sequenceintrinsic information, protein-protein datasets and orthologous groups. Gexplora is available under https://github.com/nthomasCUBE/Gexplora.
\end{abstract}

Keywords: Visualization tool, Ensembl, OMA, STRING database, REST API, genome analysis. 


\section{Introduction}

In the last years we observed a steady increase in the number of sequenced genomes granted by improvements and release of sequencing technologies, assembly strategies that consider shorter and longer reads, hybrid assemblies ${ }^{12}$ and optical maps to merge scaffolds

1. Other technical aspects include the improvements of computational tools for read assembly ${ }^{11}$ and strategies to filter reads prior to analysis ${ }^{13}$ as well as approaches to perform gap-filling ${ }^{14}$. This along with reductions in sequencing costs and the requirement to focus more intensively on the biology of a novel genome - as sequencing has become feasible for many research groups - has led to a shift from single genome focussed analysis towards analysis of meta-genomes and pan-genomes ${ }^{2}$ and thereby allowed to draw a broader picture that explains genomic or transcriptional differences between cultivars, wild types or subpopulations.

A relevant example for a large and challenging genome is bread wheat (Triticum aestivum) where the genome size exceeds the human genome by fivefold. Thereby, in the last years, research consortia obtained great success in determining the draft genome and later its near-complete linear genomic template ${ }^{3}$. In case that a genome was successfully assembled into chromosomes, underlying datasets are typically deposit in central repositories such as Ensembl ${ }^{4}$ or Orthologous Matrix Database ${ }^{5}$ which enable a collection of genomes or gene annotations from multiple species while in the field of microbes frequently used resources include eggNOG 9 and NCBI Microbial Genomes (https://www.ncbi.nlm.nih.gov/genome/microbes/).

The analysis of genome structure and gene content is key to assess the completeness or relatedness of a genome of interest and there exist plenty tools to compare the density of genes overall, e.g. the decay of orthologous genes or to derive syntenic blocks that can be then visualized with tools such as Circos ${ }^{6}$ or CrowsNest ${ }^{7}$, among many other supportive tools which help to understand the evolution of the species when facing introgression events or when related genomes are compared to a common ancestor.

In order to analyse the density of candidate and gene density in crops, in a previous study, we have implemented chromoWIZ ${ }^{10}$ as a web service to visualize gene densities along their genomes with a focus on four selected plant genomes namely Brachypodium distachyon, Hordeum vulgare, Triticium aestivum and Oryza sativa. In the current version, we provide more features with the possibility to consider any genome where a gene transfer formatted (GTF) file is available as well as selected interfaces to public databases such as OMA, STRING database and Ensembl that account for the most commonly used web resources for obtaining and analyzing genomic data. 
In the last two decades' genomics and genetic resources were mainly accessible by the institutes who coordinated the study including a FTP repository and ideally web forms to query the data. However, a comparison between different datasets was often difficult due to slightly different data standards. Meanwhile, a more efficient and common way to provide genomic datasets is to share and integrate data files between tools on the basis of REpresentational State Transfer API (REST API) ${ }^{8}$ calls that often provide JSON-formatted methods ('endpoints') that allow to request ('GET), update ('PUT') or send data ('POST'). Many resources provide such endpoints which can be then integrated into customized tools or scripts. To demonstrate this for the analysis of gene distributions, we used selected endpoints from the three resources: Gexplora integrates endpoints from Ensembl in order to obtain sequence-based information while we used OMA to retrieve more information about orthologous proteins and string-DB to determine associated publications and protein-protein interactions.

\section{Material and Methods}

\section{Calculation of the density of genetic elements}

The distribution of each element type (e.g. gene or mRNA) can be selected after the GTF (gene transfer format) file has been uploaded into Gexplora. The density of an element type is shown per bin which represents one visualization unit and indicates 1/200 of the chromosome length by considering the largest genome. Bins, with the highest amount of elements are shown in red while the low amounts of elements are shown in blue. Gexplora depicts the first five chromosomes. For genomes with more chromosomes, the next five chromosomes can be shown by activating 'forward' or 'reverse' buttons. The maximum density can be set by the user in the top panel of the tool. If the density of the candidate genes should be shown, this is possible via the option 'Find gene' allowing to upload the candidate genes. The graphical user interface Gexplora is implemented using the package 'Tkinter' package that is available in the default python installation while several additional python packages including numpy, requests and json to parse the response objects from three resources. For the export of the densities per chromosomes we make use of the Python package $X I s x W r i t e r{ }^{16}$. This can be done with the executable 'pip.exe' in Windows that is located in the 'Scripts' package of the Python installation directory. For the graphical user interface the Python version 3.7 was used along with Windows as the operating system.

\section{Obtaining data through REST API calls}

We have used three external databases that provide REST APIs: Ensembl, STRING database and OMA Orthology Database (Figure 1). For Ensembl, we allow to obtain 
sequences for individual genes (entrypoint: /family/member/id/<gene $>$ ). From OMA we obtain

the gene family that contains the candidate gene (method: orthologs) while STRING database allows to retrieve publications that are associated with the gene (abstractsList).

\section{Extraction of the data described in the show cases}

We have downloaded the GTF file from Ensembl (ftp://ftp.ensemblgenomes.org/pub/release46/plants/gff3/brachypodium_distachyon).

\section{Results}

In our last study that we have published several years ago ${ }^{10}$, we have described the webserver chromoWIZ to display gene distributions along four selected and agriculturally important or model plants namely Brachypodium distachyon, Triticum aestivum, Hordeum vulgare and Oryza sativa. As the analysis of the density of element types might be also of interest for many other genomes or genomes where annotation files are regularly updated, we provide most of the features also in Gexplora within a graphical user interface with the option to change the annotation file (see Table 1, Figure 1). These annotation files can be e.g. downloaded from Ensembl. Apart from the basic functions to visualize the element density, we offer export functions (e.g. statistics of the amount of elements per chromosomes) and interfaces to three commonly used web repositories Ensembl, OMA and STRING database.

While OMA allows to analyse orthologous proteins in comparison to other species; STRING database allows to analyse protein-protein interactions as well as provides literature entries that reference a gene among many other features. The REST API of Ensembl we have used to retrieve a gene tree for a candidate gene. This can be helpful, when a researcher wants to obtain the orthologous genes, e.g. compare whether there are differences in the copy numbers compared to other closely or distantly related species. This is provided by two endpoints as provided by Ensembl using REST API calls.

One of the big challenges in using different genomic web repositories is that gene identifiers change frequently. Otherwise, genomic repositories have their own gene identifiers. For instance, OMA uses its own gene identifiers, while STRING database uses public gene identifiers and Ensembl uses its own identifiers as well. To allow comparison and integration of the tools, mapping of identifiers is crucial. Fortunately, all these resources also provide the gene names, which was a central feature in Gexplora to allow comparison where a user can enable to also search using the gene name. 


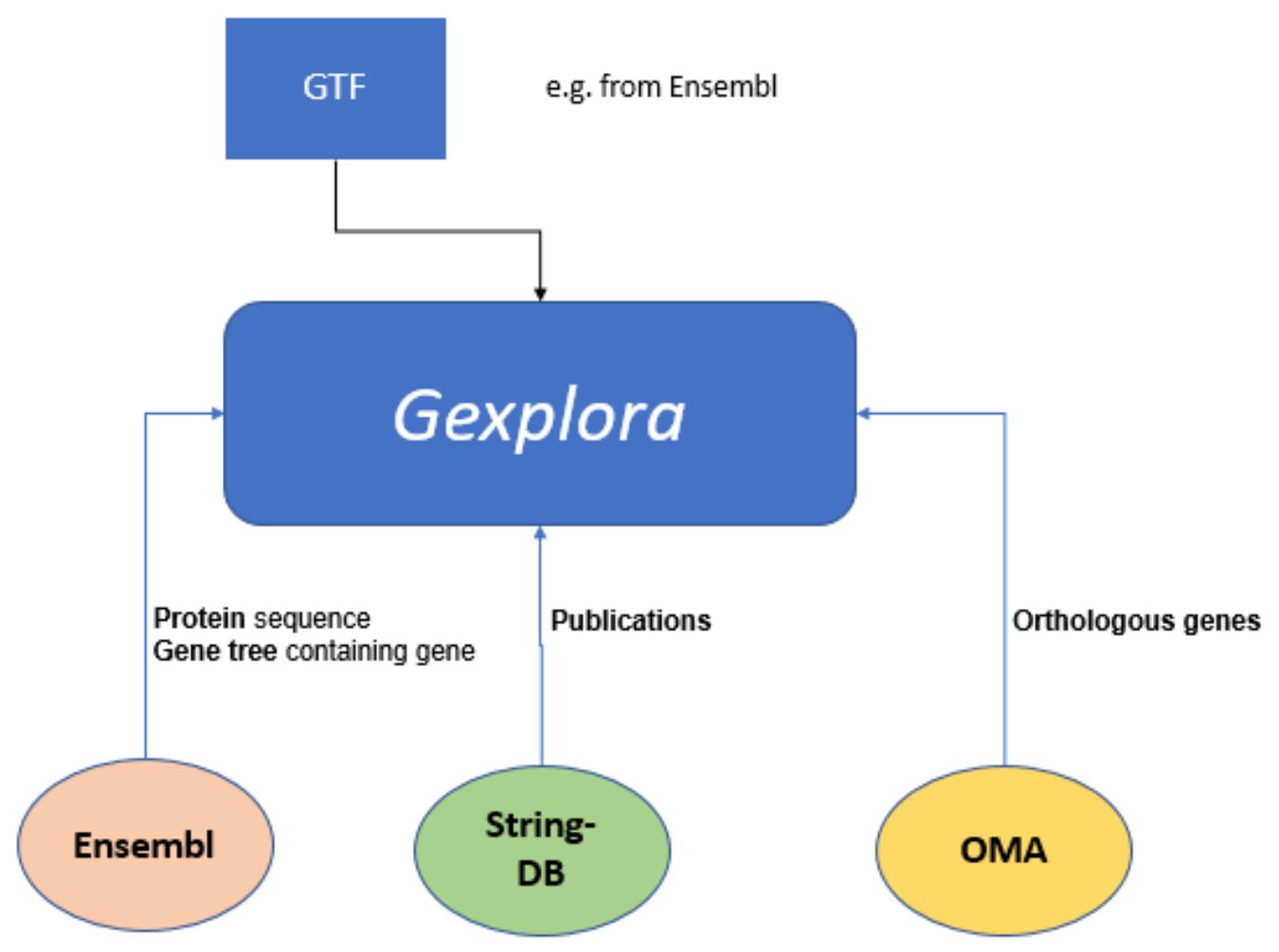

Figure 1. Gexplora workflow. The workflow illustrates the functionality of Gexplora where a user can upload a gene transfer file (GTF) as input and where interfaces to Ensembl, STRING database and OMA exist.

\begin{tabular}{|l|l|l|}
\hline Aspect & chromoWIZ & Gexplora \\
\hline Species to analyse & $\begin{array}{l}\text { Brachypodium distachyon, Triticum } \\
\text { aestivum, Hordeum vulgare, Oryza } \\
\text { sativa }\end{array}$ & $\begin{array}{l}\text { any genome with a GTF (gene transfer } \\
\text { format) files of genetic elements }\end{array}$ \\
\hline Features & $\begin{array}{l}\text { Mapping of genes to chromosomes } \\
\text { Enrichment analysis for all } \\
\text { chromosomes whether contain more } \\
\text { genes than expected }\end{array}$ & $\begin{array}{l}\text { Mapping of genes to chromosomes } \\
\text { (Enrichment analysis whether genes are } \\
\text { enriched in certain chromosomes) }\end{array}$ \\
\hline Integration to Ensembl & n.a. & $\begin{array}{l}\text { Retrieval of sequences and gene trees } \\
\text { of one or several candidate genes with } \\
\text { Ensembl REST API } \\
\text { Orthologous genes with OMA } \\
\text { Pubmed ldentifiers referencing a } \\
\text { candidate gene with string-DB }\end{array}$ \\
\hline
\end{tabular}

Table 1. Overview of the various tools and when compared to the previous version of the tool. 


\section{Use case - Analysing the model plant Brachypodium distachyon}

To benchmark our tool, we used the annotation of Brachypodium distachyon that contains five chromosomes. After the annotation file was uploaded, various element types appear (e.g. mRNA, tRNA) and can be selected. Upon selection, this triggers redrawing the overall density of the selected element type (e.g. ' $m R N A$ ' in Figure 1). We also offer an upload of candidate genes where after upload, the tool depicts the distribution of candidate genes along the chromosome using the file upload that is provided in 'Find genes' (Figure 2). For particular genes, we allow to obtain the coding sequences from Ensembl as well as a gene tree that contains orthologous and homologous genes to closely related species (Figure 2BC). By considering the STRING database, we are able to search for literature referencing these genes where candidate genes are described while the OMA interface allows to obtain all orthologous proteins.

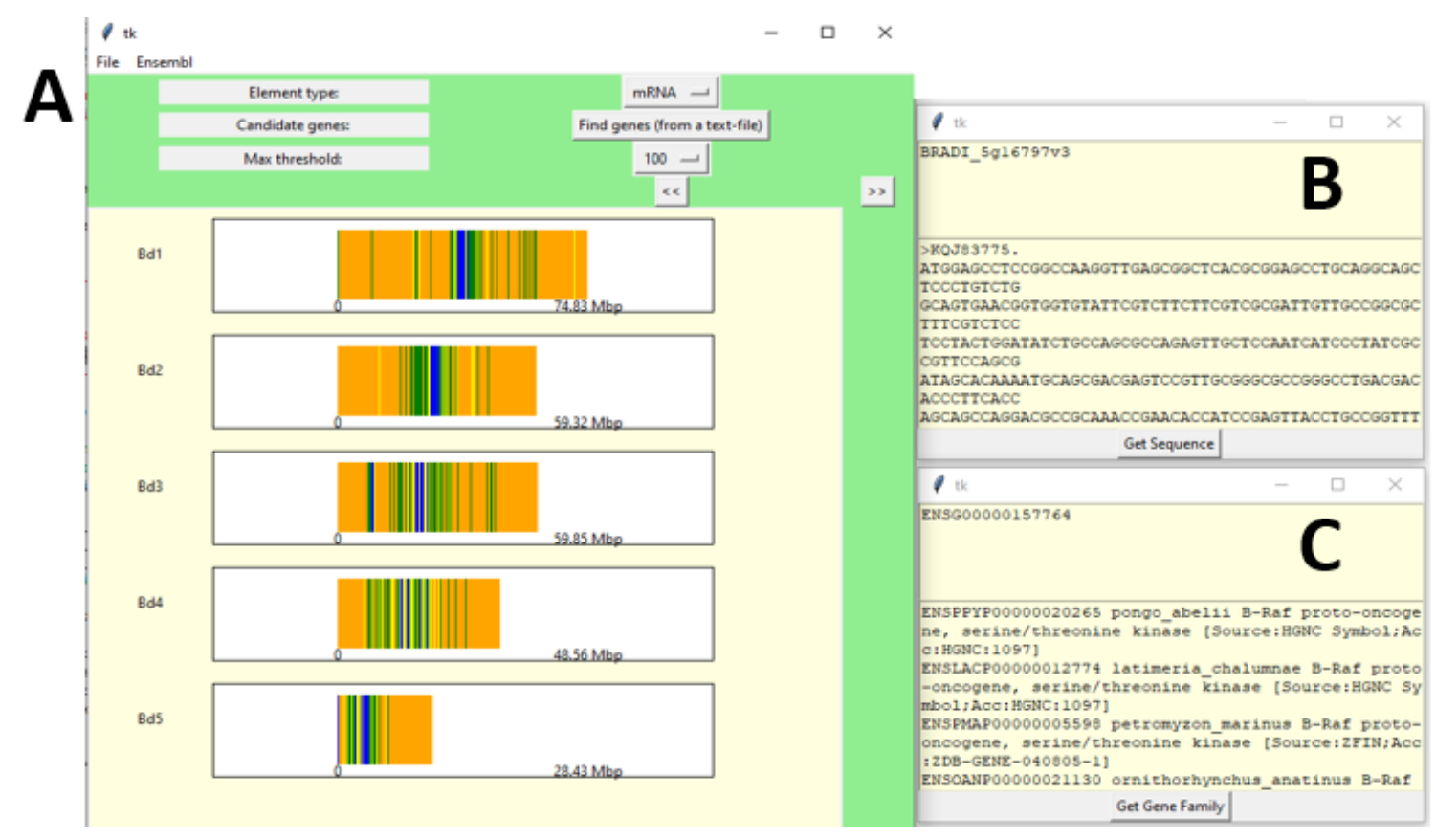

Figure 2. Gexplora. Gexplora takes an annotation file in GTF (gene transfer format) file as input (File -> Load). Main front end is shown in subfigure A, while subfigure B shows the coding sequence of a candidate gene as a result from an Ensembl search while subfigure $C$ shows all genes that share homology to the given human candidate genes using Ensembl. 


\section{Conclusion}

Gexplora as a user-friendly interface tool provides a global overview of genomic elements of candidate genes using Ensembl, STRING database, and OMA and allows to display the density of genetic elements but also various features to obtain information of orthologous genes and sequences from three databases. As these databases offer many more methods and because we provide the source code of the tool, the interfaces can be adapted easily.

\section{Availability}

Gexplora is available under: https://github.com/nthomasCUBE/Gexplora.

\section{References}

1. Zhu, T., Wang, L., Rodriguez, J. C., Deal, K. R., Avni, R., Distelfeld, A., ... \& Luo, M. C. (2019). Improved genome sequence of wild emmer wheat Zavitan with the aid of optical maps. G3: Genes, Genomes, Genetics, 9(3), 619-624.

2. Sherman, R. M., Forman, J., Antonescu, V., Puiu, D., Daya, M., Rafaels, N., ... \& Levin, A. M. (2019). Assembly of a pan-genome from deep sequencing of 910 humans of African descent. Nature genetics, 51(1), 30-35.

3. Zimin, A. V., Puiu, D., Hall, R., Kingan, S., Clavijo, B. J., \& Salzberg, S. L. (2017). The first near-complete assembly of the hexaploid bread wheat genome, Triticum aestivum. Gigascience, 6(11), gix097.

4. Zerbino, D. R., Achuthan, P., Akanni, W., Amode, M. R., Barrell, D., Bhai, J., ... \& Gil, L. (2018). Ensembl 2018. Nucleic acids research, 46(D1), D754-D761.

5. Altenhoff, A. M., Glover, N. M., Train, C. M., Kaleb, K., Warwick Vesztrocy, A., Dylus, D., ... \& Redestig, H. (2018). The OMA orthology database in 2018: retrieving evolutionary relationships among all domains of life through richer web and programmatic interfaces. Nucleic acids research, 46(D1), D477-D485.

6. Krzywinski, M., Schein, J., Birol, I., Connors, J., Gascoyne, R., Horsman, D., ... \& Marra, M. A. (2009). Circos: an information aesthetic for comparative genomics. Genome research, 19(9), 1639-1645.

7. Spannagl, M., Nussbaumer, T., Bader, K., Gundlach, H., \& Mayer, K. F. (2017). PGSB/MIPS PlantsDB database framework for the integration and analysis of plant genome data. In Plant Genomics Databases (pp. 33-44). Humana Press, New York, NY.

8. Madduri, R. K., Sulakhe, D., Lacinski, L., Liu, B., Rodriguez, A., Chard, K., ... \& Foster, I. T. (2014). Experiences building Globus Genomics: a next-generation sequencing analysis service using Galaxy, Globus, and Amazon Web Services. Concurrency and Computation: Practice and Experience, 26(13), 2266-2279.

9. Szklarczyk, Damian, et al. "The STRING database in 2017: quality-controlled protein-protein association networks, made broadly accessible." Nucleic acids research (2016): gkw937.

10. Nussbaumer, Thomas, et al. "chromoWIZ: a web tool to query and visualize chromosomeanchored genes from cereal and model genomes." BMC plant biology 14.1 (2014): 348.

11. Trapnell, Cole, Lior Pachter, and Steven L. Salzberg. "TopHat: discovering splice junctions with RNA-Seq." Bioinformatics 25.9 (2009): 1105-1111.

12. Leung, Henry CM, Siu-Ming Yiu, and Francis YL Chin. "IDBA-MTP: a hybrid metatranscriptomic assembler based on protein information." International Conference on Research in Computational Molecular Biology. Springer, Cham, 2014. 
bioRxiv preprint doi: https://doi.org/10.1101/2020.04.04.025379; this version posted April 5, 2020. The copyright holder for this preprint (which was not certified by peer review) is the author/funder, who has granted bioRxiv a license to display the preprint in perpetuity. It is made available under aCC-BY-NC-ND 4.0 International license.

13. Kopylova, Evguenia, Laurent Noé, and Hélène Touzet. "SortMeRNA: fast and accurate filtering of ribosomal RNAs in metatranscriptomic data." Bioinformatics 28.24 (2012): 32113217.

14. Boetzer, Marten, and Walter Pirovano. "Toward almost closed genomes with GapFiller." Genome biology 13.6 (2012): R56.

15. Donlin, Maureen J. "Using the generic genome browser (GBrowse)." Current Protocols in Bioinformatics 28.1 (2009): 9-9.

16. XlsxWriter. https://xlsxwriter.readthedocs.io/. Last accessed: $4^{\text {th }}$ april 2020. 